\title{
Los implantes de titanio con superficie grabada con ácidos. Un seguimiento clínico a 2 años
}

\author{
VELASCO ORTEGA E* \\ MEDEL SOTERAS R** \\ LINARES GANCEDO $\mathbf{D} * *$ \\ MONSALVE GUIL $L * *$ \\ VELASCO PONFERRADA C**
}

\begin{abstract}
Velasco Ortega E, Medel Soteras R, Linares Gancedo D, Monsalve Guil L, Velasco Ponferrada C. Los implantes de titanio con superficie grabada en ácidos. Un seguimiento clínico a 2 años. Av Periodon Implantol. 2004; 16, 3: 179-186.
\end{abstract}

\begin{abstract}
RESUMEN
Introduccion. La tecnologia implantológica está mejorando la experiencia clínica de los implantes oseointegrados, con nuevos diseños y composición en su superficie. El estudio muestra la evaluación de los implantes oseointegrados de titanio con superficie grabada con ácidos y carga precoz en pacientes con pérdida dental unitaria, parcial y total.

Métodos. 35 pacientes fueron tratados con 100 implantes TSA Defcon ${ }^{\circledR}, 53$ en la mandíbula y 47 en el maxilar superior. Todos los implantes fueron insertados en 1 fase quirúrgica. Los implantes fueron cargados funcionalmente tras un periodo de tiempo de 6 semanas (mandíbula) y de 8 semanas (maxilar superior). Los hallazgos clínicos (implantológicos y prostodóncicos) se han seguido durante 2 años.
\end{abstract}

Resultados. Tras la cicatrización (6-8 semanas), 19 pacientes fueron restaurados con coronas unitarias $(54,3 \%), 9$ pacientes con sobredentaduras $(25,7 \%)$ y 7 pacientes con puentes fijos (20\%), respectivamente. Se observaron complicaciones precoces, durante el periodo de cicatrización libre de carga funcional, en 3 implantes que fueron extraidos por movilidad. Estos resultados indican una supervivencia y éxito de los implantes, de $97 \%$.

Conclusiones. Los hallazgos clínicos del presente estudio sugieren que la utilización de los implantes de titanio con superficie grabada al ácido pueden obtener la oseointegración y ser cargados precozmente, representando un procedimiento técnico con éxito en los pacientes con pérdida de dientes.

\section{PHLABRĀS CLAVE}

Implantes orales, superficie grabada con ácidos, oseointegración, carga precoz, prótesis implantosoportadas, implantología oral.

Aceptado para publicación: Marzo 2004

\section{INTRODUCCIÓN}

La implantología oral ha revolucionado la práctica de la odontología durante los últimos 15 años. Múltiples estudios han demostrado que la rehabilitación de los pacientes desdentados parciales y totales con implantes dentales es un procedimiento predecible (1-3). En este sentido, el pronóstico a largo plazo de la terapéu-

* Profesor Titular de Odontología Integrada de Adultos y Gerodontología. Facultad de Odontología. Universidad de Sevilla.

** Profesor Colaborador Docente de Gerodontología. Facultad de Odontología. Universidad de Sevilla. 
tica con implantes está relacionado con el desarrollo del fenómeno de la oseointegración y de una buena reacción de los tejidos mucoperimplantarios (4-5).

Desde el comienzo, diversos sistemas de implantes oseointegrados se han utilizado en la rehabilitación oral del paciente parcial y totalmente desdentado lo que ha representado un reto permanente en el desarrollo de nuevos materiales y diseños, tanto en los componentes propiamente implantológicos como en los aditamentos prostodóncicos. El material más frecuentemente utilizado en la realización de los implantes orales ha sido el titanio comercialmente puro, debido a que presenta una gran biocompatibilidad y constituye el material ideal para conseguir la oseointegración con éxito a largo plazo tras la carga funcional (6-7).

La superficie de los implantes constituye un aspecto importante de la implantología oral porque sus características físicas y químicas pueden influir en el desarrollo de una mejor respuesta tisular del huésped a los implantes (8). Los implantes oseointegrados están disponibles con varias superficies desde unas relativamente lisas hasta unas superficies rugosas desarrolladas por aposición de titanio (TPS, titanium plasma spray), chorreado de arena (sandblasted), grabado ácido (acid-etched surface) o por la combinación de varios tratamientos (9).

Los estudios in vitro demuestran el interés de los investigadores por los diferentes tratamientos sobre la superficie de los implantes que pueden tener futuras implicaciones en la práctica clínica (10). En este sentido, un estudio reciente demuestra que la superficie tratada con chorreado de arena y grabado ácido que incrementa la rugosidad del implante puede mejorar la adhesión de células de estirpe osteoblástica y tener un efecto sobre la configuración y conformación de pseudópodos celulares lo que aumentaría la proliferación celular a la superficie del implante (11).

Diversos estudios in vivo realizados en animales de experimentación han demostrado que la oseointegración puede facilitarse por el incremento de la rugosidad de la superficie de los implantes (12-16). En este sentido, el torque de las fuerzas de remoción ha sido usado como una medida biomecánica del control de anclaje u oseointegración en la cual las fuerzas necesarias para extraer implantes roscados pueden ser interpretadas como un incremento en la oseointegración (9). Klokkevold et al 12 refieren, en un estudio realizado sobre el fémur de conejos (tras 8 semanas desde su inserción), que el grabado ácido de la superficie de los implantes multiplica por 4 veces la fuerza de la oseointegración determinada por la resistencia a la rotación inversa del torque en comparación con implantes de titanio de superficie pulida. Abrahamson et al 16 realizan un estudio con implantes de 2 superfi- cies diferentes en la mandíbula de perros beagle, demostrando que después del periodo de cicatrización de 3 meses, y tras otros 3 meses con los pilares conectados, existe un mayor grado de contacto entre el hueso y el implante en aquellos con superficie grabada con 2 ácidos que los de superficie pulida.

Así mismo, la experiencia clínica demuestra que los implantes con una superficie rugosa pueden obtener una mejor oseointegración y mayor tasa de éxito que los implantes de superficie lisa (17). En la última década, diversos estudios clínicos han demostrado la utilidad de los diversos tratamientos de la superficie de titanio de los implantes en el tratamiento de los pacientes con pérdida dental parcial y total con un éxito superior al 95\% (18-21). De Leonardis et al (18) expresan que la oseointegración puede ser un resultado predecible tras el seguimiento durante 5 años de 100 implantes Minimatic con superficie grabada con ácido en 63 pacientes edéntulos parciales con un éxito del $98 \%$. Velasco et al (20) en un estudio español evalúan los resultados clínicos de la utilización de 155 implantes no sumergidos ITI recubiertos de plasma de titanio en 73 pacientes con pérdida dental unitaria, parcial y total, demostrando un éxito del $95 \%$, con un seguimiento medio de 39,6 meses. Finalmente, Testori et al (21), reportan los resultados de un estudio múlticéntrico con 485 implantes con superficie grabada con ácido (Osseotite) después de un seguimiento de 4 años, con un éxito del $98,7 \%$.

En los últimos años, está siendo extraordinaria la investigación implantológica para mejorar los resultados clínicos y el éxito de los implantes oseointegrados, con nuevos diseños y composición en su superficie. El objetivo del presente estudio consiste en la valoración de los implantes oseointegrados de titanio con superficie grabada con ácidos y carga precoz en pacientes con pérdida dental.

\section{PACIENTES Y MÉTODOS}

El presente estudio fué realizado por profesores de la Facultad de Odontología de la Universidad de Sevilla. Previamente a la realización del estudio, aquellos pacientes que padecían trastornos sistémicos graves que podían comprometer la oseointegración fueron excluidos del estudio (22). Los pacientes seleccionados eran adultos, de ambos sexos. Con respecto a las condiciones orales, los pacientes no presentaban enfermedad periodontal, ni historia de haberla padecido en el pasado. Un requisito importante fué la disponibilidad de una suficiente cantidad de hueso alveolar en los maxilares o tramos edéntulos ya que el presente estudio solamente incluía la realización de 
los procedimientos quirúrgicos básicos y se excluyeron las técnicas más complejas (ej. regeneración tisular, injertos óseos, factores de crecimiento).

Todos los pacientes seleccionados en el estudio fueron informados del protocolo del tratamiento con implantes, de los aspectos quirúrgicos y prostodóncicos, temporalización del tratamiento y seguimiento, así como de la posibilidad de la existencia de complicaciones y pérdidas de implantes. Los pacientes autorizaron el tratamiento implantológico mediante un consentimiento informado. Antes del tratamiento, todos los pacientes fueron evaluados radiológicamente, con una ortopantomografía, además cuando fué necesario se realizaron tomografías computarizadas y radiografías periapicales.

Los criterios de éxito y supervivencia de los implantes fueron los recomendados por van Steenberghe et al (23). En este sentido, la supervivencia fué definida como la proporción de implantes permanentes en su localización original aunque no tengan valor clínico o cause efectos adversos. Los criterios de éxito de los implantes se expresan en la Tabla 1.

CIRUGÍA. Una hora antes de la cirugía, los pacientes comenzaron un regimen antibiótico preventivo (amoxicilina + clavulánico) durante una semana. Todos los pacientes recibieron anestesia local. Un total de 100 implantes TSA Defcon ${ }^{\circledR}$ (Impladent, Sabadell, España) roscados de superficie grabada y pasivada químicamente con ácidos (fluorhídrico y nítrico), fueron insertados en una fase quirúrgica, y todos fueron estables después de la inserción. El procedimiento quirúrgico básico consistió en una incisión supracrestal media, con realización de colgajo de espesor total con exposición del hueso alveolar subyacente. La preparación del lecho y la inserción de los implantes se realizó según el protocolo estandarizado convencional con fresas consecutivas de menor a mayor diámetro, a una velocidad constante de 800 r.p.m. Los tejidos blandos fueron suturados alrededor de los tornillos de cicatrización (1 cirugía). A la semana se retiraron las suturas y los pacientes fueron instruídos sobre cuidados higiénicos orales básicos. Se reco- mendó el enjuague diario con clorhexidina durante los primeros 30 días.

PROSTODONCIA. Los pacientes edéntulos totales no usaron sus prótesis completa durante 4 semanas después de la inserción de los implantes. A los pacientes con edentulismo parcial o unitario no se le realizaron restauraciones provisionales, excepto en los casos del frente anterior. En estos casos, las restauraciones provisionales removibles fueron dentosoportadas sin contacto con el lecho implantario y libres de oclusión.

Después de 6 semanas en los implantes insertados en la mandíbula y de 8 semanas en los implantes maxilares, se realizó los diversos procedimientos prostodóncicos para la carga funcional de los implantes. El tiempo transcurrido desde la carga funcional y de seguimiento clínico de los implantes fué de 2 años.

ANÁLISIS ESTADÍSTICO. Se realizó una estadística descriptiva de los hallazgos clínicos del estudio, con referencia a las variables demograficas de los pacientes, la supervivencia, el éxito, las complicaciones y pérdidas de los implantes así como de las restauraciones prostodóncicas realizadas.

\section{RESULTADOS}

PACIENTES. 35 pacientes (23 hombres y 12 mujeres) participaron en el estudio y fueron tratados con implantes, con una edad media de 46,7 años (rango: 30-70 años). 9 edéntulos totales, 7 edéntulos parciales y 19 con pérdidas unitarias.

IMPLANT'ES. Se insertaron un total de 100 implantes TSA Defcon, lo que representa una media de 2,8 implantes por paciente. De los implantes utilizados, 55 fueron de 3,4 mm de diámetro; 45 de $4 \mathrm{~mm}$ de diámetro. Con respecto a la longitud, fueron insertados 42 implantes de $10 \mathrm{~mm}$, y 58 de $13 \mathrm{~mm}$ (Tabla 2). 47 implantes fueron insertados en el maxilar superior, mientras que 53 fueron en la mandíbula. La localiza-

\section{TABLA 1.- LOS CRITERIOS DE EXITO EN EL TRATAMIENTO CON IMPLANTES (van Steenberghe et al ${ }^{23}$ )}

1.- El implante no provoca ninguna reacción alérgica, tóxica o infecciosa de carácter local o sistémico.

2.- El implante ofrece soporte para una prótesis funcional.

3.- El implante no muestra signos de fractura o incurvación.

4.- El implante no muestra ninguna movilidad cuando es explorado manual o electrónicamente.

5.- El implante no muestra ningún signo de radiolucidez mediante una radiografía intraoral.

6. - La pérdida marginal de hueso (Rx intraoral) y/o la pérdida de inserción (profundidad de sondaje + recesión) no deben perjudicar la función de anclaje del implante o causar molestias para el paciente durante 20 años. 


\begin{tabular}{|cccc|}
\hline \multicolumn{5}{|c|}{ TABLA } & 2.- DISTRIBUCIÓN DE LOS DIVERSOS TIPOS DE IMPLANTES \\
INSERTADOS SEGÚN SU LONGITUD Y DIÄMETRO
\end{tabular}

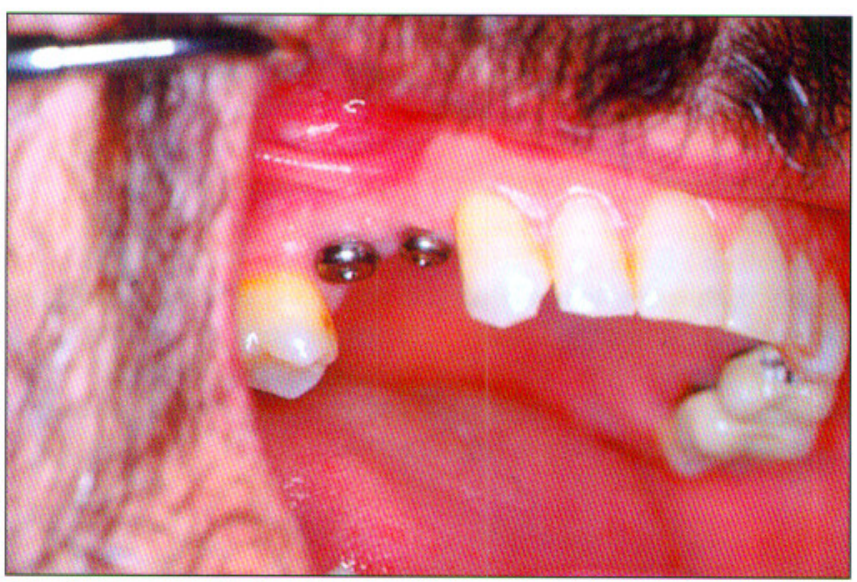

Fig. 1. Aspecto clínico del paciente con pérdida de los premolares superiores derechos y su sustitución por dos implantes no sumergidos con superficie grabada con ácidos.

ción de los implantes según el sector anterior o posterior de los maxilares se expone en la Tabla 3. Todos los implantes (100\%) fueron insertados de forma no sumergida en 1 fase quirúrgica (Figura 1).

En 3 implantes (3\%) hubo complicaciones. Los 3 implantes presentaron movilidad a la exploración clínica durante la fase de cicatrización libre de carga funcional y fueron extraídos. Ningún implante se ha perdido después de la carga funcional prostodóncica durante los 2 años de seguimiento clínico.

PRÓTESIS IMPLANTOSOPORTADAS. 97 implantes fueron cargados funcionalmente, con su correspondiente

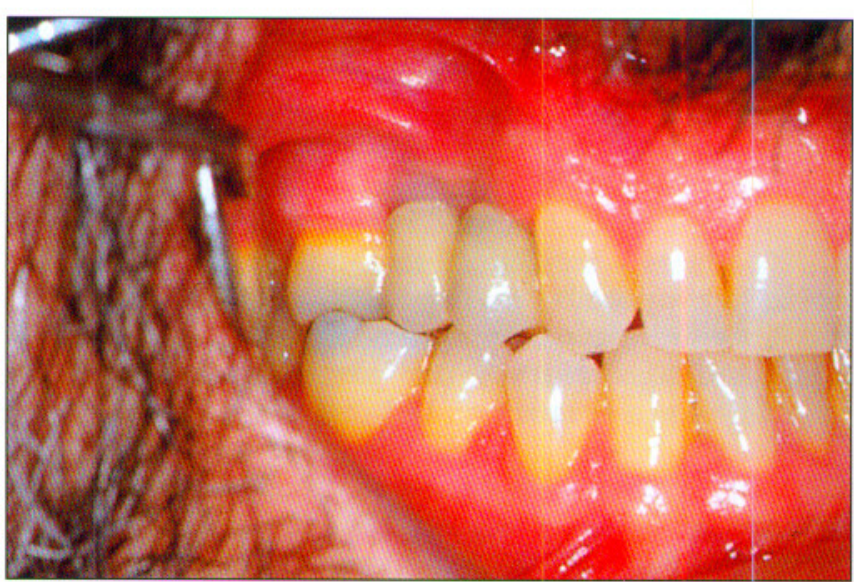

Fig. 2. Aspecto clínico del paciente con la restauración prostodóncica sobre los 2 implantes con superficie grabada con ácidos con carga precoz a los 2 meses.

restauración prostodóncica implantosoportada, 51 implantes en la mandíbula (a las 6 semanas) y 46 implantes en el maxilar superior (a las 8 semanas). Tras el periodo de oseointegración se realizaron las siguientes prótesis dentales: 37 coronas unitarias, 8 prótesis o puentes fijos ( 7 con 2 implantes y 1 con 3 implantes) (Figura 2), y 12 sobredentaduras (10 con retención mediante barras y 2 mediante bolas) (Tabla 4).

\section{DISCUSIÓN}

Los resultados de este estudio indican que los implan-

\begin{tabular}{|llll|}
\hline \multicolumn{2}{|c|}{ TABIA } & 3.- DISTRIBUCIÓN DE LOS IMPLANTES SEGÚN LA LOCALIZACIÓN & Total \\
\hline Maxilar & Anterior* & P osterior** & $47(100 \%)$ \\
\hline Superior & $22(46,8 \%)$ & $25(53,2 \%)$ & $53(100 \%)$ \\
Inferior & $27(50,9 \%)$ & $26(49,1 \%)$ & \\
\hline * Anterior: implantes situados en el sector de dientes incisivos y caninos. \\
** Posterior: implantes situados en el sector de dientes premolares y molares.
\end{tabular}




\section{TABLA 4.- PRÓTESIS DENTALES REALIZADAS SOBRE LOS IMPLANTES INSERTADOS}

\begin{tabular}{|ccr|}
\hline Prótesis Dentales & Número & $\%$ \\
\hline Coronas unitarias & 37 & 64,9 \\
Sobredentaduras & 12 & 20,1 \\
Puentes fijos & 8 & 14 \\
\hline Total & $\mathbf{5 7}$ & $\mathbf{1 0 0} \%$ \\
\hline
\end{tabular}

tes orales con superficie grabada con ácidos pueden ser insertados con éxito y obtener una oseointegración adecuada para su carga funcional prostodóncica. Este trabajo presenta los datos de la inserción de implantes orales en maxilares parcial y totalmente edéntulos, así como su posterior función en la sustitución de dientes unitarios, demostrando una supervivencia y éxito de los implantes de $97 \%$ con 2 años de seguimiento clínico. En este sentido, los favorables resultados del presente estudio pueden ser atribuidos a varias razones, a) la selección de los pacientes; b) el diseño y el material biocompatible del implante dental con una superficie de titanio grabada y pasivada con ácidos; c) el estandarizado instrumental del sistema de implantes dentales utilizado que facilita la realización del lecho implantario; y d) los aditamentos prostodóncicos para las diversas soluciones protésicas implantosoportadas.

En el presente estudio se ha valorado el éxito de la utilización de implantes con superficie grabada con ácidos. En este sentido, el tratamiento de la superficie de los implantes orales incrementado la rugosidad o desarrollando microcavidades, fisuras o grietas mediante diversas tecnologías (ej. plasma de titanio, chorreado de arena, grabado ácido) puede favorecer la unión entre las macromoléculas de la superficie del implante y del hueso, resultando en una mayor resistencia a la compresión, tensión y estrés. Se ha demostrado que una mayor rugosidad de la superficie implantaria incrementa la integración del implante con una mejor respuesta tisular osteoblástica $(8,18)$. En este estudio se demuestra que la técnica no sumergida tiene un éxito terapéutico predecible y proporciona una oseointegración favorable, aceptándose en la actualidad el éxito de ambos sistemas de implantes sumergidos y no sumergidos comercializados (1-3).

En el presente estudio, todas las complicaciones aparecieron durante el periodo de cicatrización libre de carga funcional. 3 implantes presentaron movilidad precoz. Los 3 implantes fueron extraídos. Los fracasos fueron considerados como fracasos precoces. Estos fracasos precoces suelen ser causados por el trauma quirúrgico (ej. sobrecalentamiento del lecho implantario), la falta de estabilidad primaria, la infección bacteriana y la acción de fuerzas masticatorias. Las complicaciones precoces representan la imposibilidad de establecer una adecuada reacción tisular del organismo con la superficie de los implantes, es decir no se produce la oseomucointegración, existe movilidad implantaria y el tratamiento exige la extracción del implante (24).

Además de valorar el éxito y predicibilidad en conseguir la integración tisular de los implantes utilizados, otro aspecto interesante del estudio consistía en realizar la carga precoz de los implantes con sus correspondientes prótesis dentales. De hecho, el tratamiento con ácidos de la superficie de titanio para favorecer una mayor rugosidad pretende conseguir una oseointegración favorable y más rápida que permita disminuir el tiempo de espera libre de carga funcional. En este sentido, la fase prostodóncica ha sido realizada, tras la inserción de los implantes, después de 6 semanas en la mandíbula y 8 semanas en el maxilar superior. Desde este perspectiva, los resultados prostodóncicos del presente estudio demuestran un éxito del $100 \%$ durante los 2 años transcurridos desde la carga funcional de los implantes con sus correspondientes soluciones protésicas en la terapéutica implantológica del edentulismo unitario, parcial y total.

En la actualidad, la carga precoz de los implantes constituye un reto importante en el avance y desarrollo de la implantología oral porque de la investigación científica experimental y clínica se están beneficiando directamente, cada vez más, los pacientes con pérdida dental (25-26). Ahora bien, además de la superficie tratada de los implantes, los protocolos de carga precoz deben comprender unos aspectos quirúrgicos muy estrictos, una selección cuidadosa de los pacientes con una buena calidad y cantidad de hueso, una estabilidad primaria de los implantes con una reducción máxima de los micromovimientos para impedir la fibrointegración, y finalmente -pero no por eso menos importante- un diseño adecuado de las soluciones protésicas implantosoportadas que cumplan los requisitos imprescindibles de la una oclusión funcional (27).

Generalmente, los implantes son utilizados para el tratamiento de los pacientes con maxilares parcial y 
totalmente edéntulos. Sin embargo, en la actualidad, se ha incrementado el número de implantes unitarios. En el presente estudio, 37 implantes unitarios con superficie grabada con ácidos fueron cargados precozmente con sus correspondientes coronas en el $54,3 \%$ de los pacientes tratados. De hecho, los estudios clínicos demuestran que los implantes unitarios ofrecen unos resultados excelentes con un éxito entre el $95-100 \%(2,28)$. Este éxito puede deberse a la presencia de los dientes contiguos y un adecuado soporte óseo que limita un excesiva carga oclusal por lo que pueden ser cargados precozmente, como demuestran Cooper et al 25 al valorar el seguimiento durante 1 año de 53 implantes unitarios Astra Tech insertados, con 1 cirugía en 52 pacientes con dientes anterosuperiores perdidos, y cargados precozmente a las 3 semanas con un éxito del $96,2 \%$.

En la actualidad, muchos pacientes que solicitan tratamiento con implantes presentan un edentulismo parcial. En el presente estudio, se han realizado 8 puentes fijos implantosoportados en el $20 \%$ de los pacientes tratados (Figuras 1 y 2). En el sector anterior, las fuerzas oclusales son menores y el volumen de hueso suele ser mayor que en las zonas posteriores, por lo que se puede reducir el número de implantes por algunos de mayor longitud, además en el sector anterior, la estética es importante, lo que exige un tratamiento adecuado de los tejidos que favorezca un aspecto lo más natural posible a la prótesis fija. En la región posterior las fuerzas oclusales son elevadas, por lo que son necesarios más implantes, si es posible de mayor diámetro y que soporten los puentes fijos en posición medial y distal con pónticos intermedios y sin extremos libres (29).

El presente estudio indica que la carga precoz de los implantes oseointegrados con grabado ácido constituye una terapéutica eficaz en la rehabilitación protésica del paciente edéntulo total. 9 pacientes edéntulos totales $(25,7 \%)$ fueron tratados con 12 sobredentaduras, 9 en la mandíbula y 3 en el maxilar superior. La experiencia clínica revela un elevado éxito en el tratamiento con sobredentaduras implantosoportadas (90-95\%) (30). Las tendencias actuales indican que la sobredentadura mandibular constituye una terapéutica eficaz con éxito en la carga precoz de de los implantes orales como demuestran Payne et al (26) al evaluar la eficacia de los implantes oseointegrados para soportar sobredentaduras mandibulares con retención mediante bolas con 2 implantes ITI SLA y comparar los resultados en 2 grupos de pacientes ( $n=12$ y $n=12$ ), dónde la carga funcional se realizó a las 12 semanas y a los 6 semanas, respectivamente, con unos resultados de éxito del $100 \%$ en ambos grupos después de 2 años de seguimiento.

\section{CONCLUSIONES}

La implantología oral ha provocado un extraordinario impacto positivo en la práctica odontológica al ofrecer un tratamiento predecible y con éxito en la rehabilitación del edentulismo parcial y total. Esta técnica basada en la respuesta tisular favorable -muco y oseointegración- a la inserción de los implantes orales unida a la experiencia investigadora y clínica actual en relación a los diversos tratamientos de la superficie de titanio (ej. grabado con ácidos) y de protocolos exigentes de carga precoz funcional está impulsado el tratamiento implantológico con un mayor beneficio de los pacientes. En este sentido, los resultados del presente estudio indican que los implantes oseointegrados con superficie grabada con ácidos pueden ser utilizados con éxito y cargados precozmente para la restauración prostodóncica de los pacientes con pérdida dental.

\section{ABSTRACT}

Introduction. Implant technology is improving the clinical experience of osseointegrated implants with new designs and compositions of their surfaces. This study reports the results of evaluation of titanium implants with acid-etched surface and early loading in patients with unitary, partially and fully tooth loss.

Methods. 100 TSA Defcon ${ }^{\circledast}$ implants were inserted in 35 patients. 53 implants were placed in the mandible and 47 in the maxilla. All implants were inserted in one-stage. Implants were loaded after a healing free-loading period between 6 weeks (mandible) and 8 weeks (maxilla). Clinical findings (implants and prosthodontics) were followed during 2 years.

Results. Following healing (6-8 weeks), 19 patients were restored with single crowns $(54,3 \%), 9$ patients with overdentures $(25,7 \%)$ and 7 patients with fixed bridges $(20 \%)$, respectively. Early complications (during the healing period) were reported in 3 implants that were removed by mobility. These results indicate a survival and success rate of implants of $97 \%$.

Conclusions. Clinical findings of this study suggest that use of etchet-surface titanium implants can achieve osseointegration and were loaded early as a successful treatment of patients with tooth loss. 


\section{KEY WORDS}

Oral implants, acid-etched surface, osseointegration, early loading implants-supported prosthetics, implant dentistry.

\section{CORRESPONDENCIA}

Dr. Eugenio Velasco Ortega

C/ José Laguillo $n^{\circ} 18,2^{\circ}-6$

Tfno: 954421805

e-mail: evelasco@us.es

41003 Sevilla

\section{BIBLIOGRAFÍA}

1. Buser D, Mericske-Stern RD, Bernard JP, et al. Long-term evaluation of non-submerged ITI implants. Part 1: 8-year life table analysis of a prospective multi-center study with 2359 implants. Clin Oral Impl Res 1997; 8: 161-72.

2. Henry PJ. Clinical experiences with dental implants. Adv Dent Res 1999; 13:147-52.

3. Noack N, Willer J, Hoffmann J. Long-term results after placement of dental implants: longitudinal study of 1.964 implants over 16 years. Int J Oral Maxillofac Implants $1999 ; 14: 748-55$.

4. Davies JE. Mechanisms of endosseous integration. Int J Prosthodont 1998; 11:391-401.

5. Weber HP, Cochran DL. The soft tissue response to osseointegrated dental implants. J Prosthet Dent 1998; 79: 7989.

6. Brunski JB, Puleo DA, Nanci A. Biomaterials and biomechanics of oral and maxillofacial implants: current status and future developments. Int J Oral Maxillofac Implants 2000; 15: 15-39.

7. Taylor TD, Agar JR, Vogiatzi T. Implant prosthodontics: current perspective and future directions. Int J Oral Maxillofac Implants 2000; 15: 66-75.

8. Kasemo B, Gold J. Implant surfaces and interface processes. Adv Dent Res 1999; 13: 8-20.

9. Sykaras N, Iacopino M, Marker VA, Triplett RG, Woody $\mathrm{RD}$. Implant materials, designs, and surface topographies: their effect on osseointegration. A literature review. Int J Oral Maxillofac Implants 2000; 15: 675-90.
10. Lim YJ, Oshida Y, Andres CJ, Barco MT. Surface characterization of variously treated titanium materials. Int J Oral Maxillofac Implants 2001; 16: 333-42.

11. Orsini G, Assenza B, Scarano A, Piattelli M, Piattelli A. Surface analysis of machined versus sandblasted and acid-etched titanium implants. Int J Oral Maxillofac Implants 2000; 15: 779-84.

12. Klokkevold PR, Nishimura RD, Adachi M, Caputo A. Osseointegration enhanced by chemical etching of the tittanium surface: a torque removal study in the rabitt. Clin Oral Impl Res 1997; 8: 442-7.

13. Buser D, Nydegger T, Hirt HP, Cochran DL, Nolte LP. Removal torque values of titanium implants in the maxilla of miniature pigs. Int J Oral Maxillofac Implants 1998; 13: 611-9.

14. Baker D, London RM, O'Neal R. Rate of pull-out strength gain of dual-etched titanium implants: a comparative study in rabbits. Int J Oral Maxillofac Implants 1999; 14: 722-8.

15. Cordioli G, Majzoub Z, Piatelli A, Scarano A. Removal torque and histomorphometric investigation of 4 different titanium surfaces: an experimental study in the rabbit tibia. Int J Oral Maxillofac Implants 2000; 15: 668-74.

16. Abrahamsson I, Zitzmann NU, Berglundh T, Wennerberg $\mathrm{A}$, Lindhe J. Bone and soft tissue integration to titanium implants with different surface topography: an experimental study in the dog. Int J Oral Maxillofac Implants 2001; 16: 323-32.

17. Cochran DL. A comparison of endosseous dental implant surfaces. J Periodontol 1999; 70: 1523-39.

18. De Leonardis D, Garg AK, Pecora GE. Osseointegration of rough acid-etched titanium implants: 5-year follow-up of 100 Minimatic implants. Int J Oral Maxillofac Implants 1994; 14: 384-91.

19. Sullivan DY, Sullivan DY, Sherwood RL, Mai TN. Preliminary results of a multicenter study evaluating a chemically enhanced surface for machined commercially pure titanium implants. J Prosthet Dent 1997; 78:379-86.

20. Velasco E, Martinez-Sahuquillo A, Machuca G, Bullón P. La valoración clínica y el seguimiento de los implantes no sumergidos ITI-Bonefit. RCOE 1998; 3: 211-6.

21. Testori T, Wiseman L, Woolfe S, Porter SS. A prospective multicenter clinical study of the Osseotite implant: fouryear interim report. Int J Oral Maxillofac Implants 2001; 16: 193-200.

22. Buser D, von Arx T, ten Bruggenkate C, Weingart D. Basic 


\section{AVANCES}

Volumen $16-\mathrm{N}^{\circ} 3$ - Diciembre 2004

surgical principles with ITI implants. Clin Oral Impl Res 2000; 11 (suppl 1): 59-68.

23. van Steenberghe D, Quirynen, Naert I. Survival and success rates with oral endosseous implants. En: Lang NP, Karring T, Lindhe J (eds.). Proceedings of the 3rd European Workshop on Periodontology. Implant Dentistry. Berlin: Quintessence. 1999. pag: 242-52.

24. Esposito M, Hirsch JM, Lekholm U, Thomsen P. Differential diagnosis and treatment strategies for biologic complications and failing oral implants: a review of the literature. Int J Oral Maxillofac Implants 1999; 14: 473-90.

25. Cooper L, Felton DA, Kugelberg CF et al. A multicenter 12-month evaluation of single-tooth implants restored 3 weeks after 1-stage surgery. Int J Oral Maxillofac Implants 2001; 16: 182-92.

26. Payne AGT, Tawse-Smith A, Duncan WD, Kumara R. Conventional and early loading of unsplinted ITI implant supporting mandibular overdentures. Clin Oral Impl Res 2002; 13: 603-9.
27. Szmukler-Moncler S, Piatelli A, Favero GA, Dubruille JH. Considerations preliminary to the application of early and immediate loading protocols in dental implantology. Clin Oral Impl Res 2000; 11: 12-25.

28. Wannfors K, Smedberg JI. A prospective clinical evaluation of different single-tooth restoration designs on osseointegrated implants. A 3-year follow-up of Branemark implants. Clin Oral Impl Res 1999; 10: 453-8.

29. Gotfredsen K. Treatment concepts for partially dentate. En: Lang NP, Karring T, Lindhe J (eds.). Proceedings of the 3rd European Workshop on Periodontology. Implant Dentistry. Berlin: Quintessence. 1999. pag: 408-20.

30. Batenburg RHK, Meijer HJA, Raghoebar GM, Vissink A. Treatment concept for mandibular overdentures supported by endosseous implants: a literature review. Int J Oral Maxillofac Implants 1998; 13: 539-45. 